\title{
Application of intelligent agents in channel allocation strategies for mobile networks
}

\author{
Eliane L. Bodanese and Laurie G. Cuthbert \\ Department of Electronic Engineering \\ Queen Mary and Westfield College - University of London \\ London, UK, E1 4NS - Tel: +44 (0) 2078823756 - Fax: +44 (0) 2078827997 \\ E-mail: \{e.1.bodanese, 1.cuthbert\}@elec.qmw.ac.uk
}

\begin{abstract}
Resource flexibility is one of the most important requirements in the next generation of mobile communications. Techniques are required to increase the flexibility of the network to deal with new services and the consequent new traffic profiles and characteristics. This paper investigates some of the drawbacks of fully reactive channel allocation schemes and proposes a more flexible scheme using intelligent agents that will lead to an efficient solution under moderate and heavy loads. The agent architecture adopted provides greater autonomy to the base stations and a method for allowing co-operation and negotiation between them; this autonomy and co-operation allows an increase in flexibility to deal with new traffic situations and an increase of the robustness of the network as a whole.
\end{abstract}

\section{INTRODUCTION}

Techniques for increasing the efficiency of channel usage in mobile networks are essential in order to handle the complexity of third generation mobile communications. Previous work on analogue and second generation mobile communications has led to several algorithms being proposed to maximise the channel usage and minimise the call blocking probability. Some channel assignment schemes presented in the literature [1-7] have improved the performance of the basic fixed channel assignment strategy for different traffic densities (macro/micro/pico cellular networks) over different traffic load conditions. However, most of the solutions proposed have an entirely reactive approach: the response to a series of events follows an algorithm that is prepared to react to specific situations. This limits their efficiency. Even those schemes that contain adaptive features are not ideal. Some schemes are completely centralised (which is impractical for real implementation because of computational complexity making them too slow, or because the network would become overloaded with signalling messages) or are distributed, but restricted to individual base stations which again limits their efficiency.

In order to accommodate multiple networks and services with multiple bit rates within a limited frequency band, resource flexibility is one of the most important requirements in third-generation networks. A multi-agent system is able to provide this flexibility and also is able to increase the robustness of the network by allowing negotiation when conflicts occur. This paper describes the framework we are adopting to verify the feasibility of multi-agent systems in controlling resource assignment in mobile networks. Our agent architecture is hierarchical, with a reactive part and a deliberative part. The reactive part is the lowest layer, ensuring fast response to the needs of the cells in terms of channel allocation. The deliberative part is concerned with optimisation of the allocation of channels among cells and consists of two layers: one to met the local goals of the individual base stations and another (the co-operative layer) for load balancing in the network.

We are assuming a macro-cellular scenario where base stations are not able to share information by interference measurements, but only by explicit exchange of information, and the resources are complete frequency carriers. Although the results in this paper are based on an AMPS [8] model, the concept is completely generic and the work could be extended to TDMA/CDMA. Different cell structures (such as microcellular or hierarchical) could also be included.

\section{Channel ASSignMent Strategies}

Fixed channel assignment (FCA) is too limiting for mobile networks and several strategies have been proposed to maximise frequency channel allocation and minimise call blocking probability. A detailed survey describing dynamic channel assignment (DCA) schemes and FCA-based schemes, including those with channel borrowing is given in [7]. In that paper it is noted that there is a trade-off between the implementation complexity of the channel allocation algorithms and spectrum utilisation efficiency. DCA schemes perform better under low traffic intensity; modified FCA schemes have superior performance in high traffic loads. DCA schemes use channels more efficiently and for the same blocking rate have a lower forced call termination than FCA-based schemes. However, the near-optimum channel allocation is at the expense of high overheads through its use of centralised allocation schemes. This overhead means that such schemes are not practicable for large networks.

Distributed DCA schemes with limited inter-cell communication suffer less overhead, but lead to sub-optimum allocations. Such schemes are being proposed for microcellular systems as this cell structure allows inter-cell information sharing by interference measurements and passive non-intrusive monitoring at each base station (busy/idle status of the carriers).

For macrocellular systems, where explicit communication is needed, FCA with channel borrowing offers good results and less computational complexity than DCA. However, those FCA schemes with the best results [1,2] used centralised control inside the Mobile Switching Centre (MSC). Although that is less complex than DCA schemes, there is still a need to maintain an up-to-date global knowledge of the entire mobile network, leading to a slow response and a heavy signalling load. To alleviate this problem, several authors have proposed modifications to make the schemes more distributed. One example is the 
distributed load balancing with selective borrowing scheme (D-LBSB) [6] that performs better than its centralised version [5] and also outperforms other existing schemes like direct retry [3] and CBWL [4]. The D-LBSB scheme is a distributed FCA algorithm with selective borrowing, channel locking and channel reassignment. It takes into consideration the position of the mobile users when borrowing and reassigning channels; it triggers the execution of the algorithm when the usage of the nominal channels in a cell reaches a pre-determined threshold ( $h$, when a previously cold cell becomes hot). It also controls the number of the channels to be borrowed from or lent to a cell according to the traffic load of the whole cellular network. We selected this algorithm as the basic comparison for our work.

\section{ANALYSIS OF SIMULATION RESULTS}

We have implemented our cellular model in the commercial simulation OPNET ${ }^{\mathrm{TM}}$. There are 49 cells and each cell has 10 nominal channels. The compact pattern is a 7-cell cluster with the reuse distance being three cell units. Mobile users have their own trajectories inside the mobile network. Call establishments and handoff requests are simulated as they are requested in AMPS systems. For each cell, a Poisson distribution is used to generate calls, which have an exponentially distributed duration with a mean of three minutes. Each call attempt is performed by an idle mobile inside the cell, chosen randomly. This is a more detailed simulation than those presented in the literature, which make broader assumptions. These differences need to be taken into account when comparing results.

A distributed borrowing algorithm based on D-LBSB was implemented generally following the descriptions in $[5,6]$. The distribution elements of the system were located in the base stations, the messages between base stations passing through the MSC.

We used a value of 0.2 for the cold/hot threshold $(h)$. Unlike the original algorithm where the departing region $(r)$ varies with the number of channels to be borrowed and the average density of mobile users in the cell, we chose $r$ to be constant and dependent on signal strength. Some necessary parameters for implementation have not been described fully in the references so that while our algorithm generally follows that of D-LBSB it is not exactly the same. We refer to our scheme as the distributed borrowing algorithm (BA).

To analyse the performance of the scheme, we chose the system layout with non-uniform traffic distributions of [2] as shown in Fig. 1. The number in the bottom of each cell is the cell identification; the number in the middle gives the Poisson arrival rates in calls/hour (ranging from 20 to 200calls/hour).

Fig. 2 shows the blocking rate of our BA compared with the FCA as the traffic rates increase over the load shown in Fig. 1.

Overall, BA outperforms FCA from moderate to heavy load, the maximum improvement achieved in this case is a reduction in call blocking probability by around $20 \%$. However, individual cells with higher traffic rates present a better improvement as shown in Fig. 3.

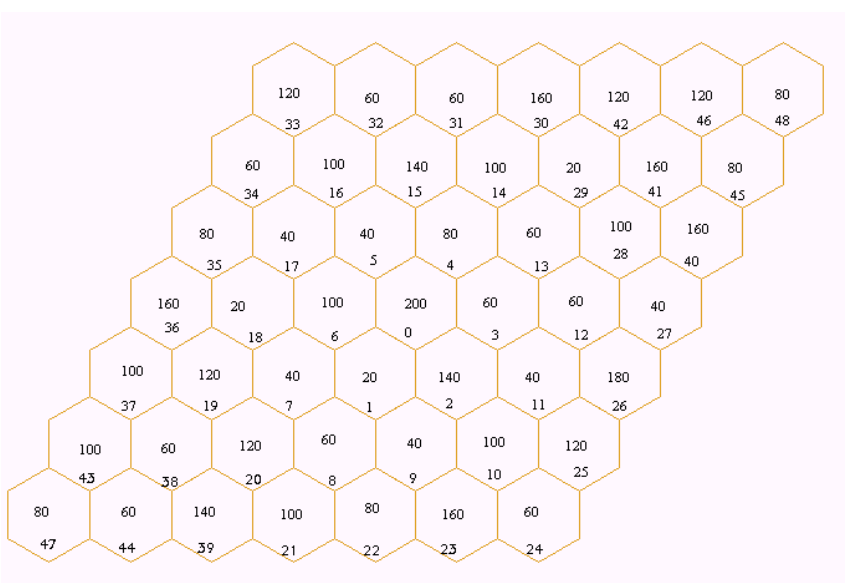

Fig. 1 Cellular system layout with non-uniform traffic distribution

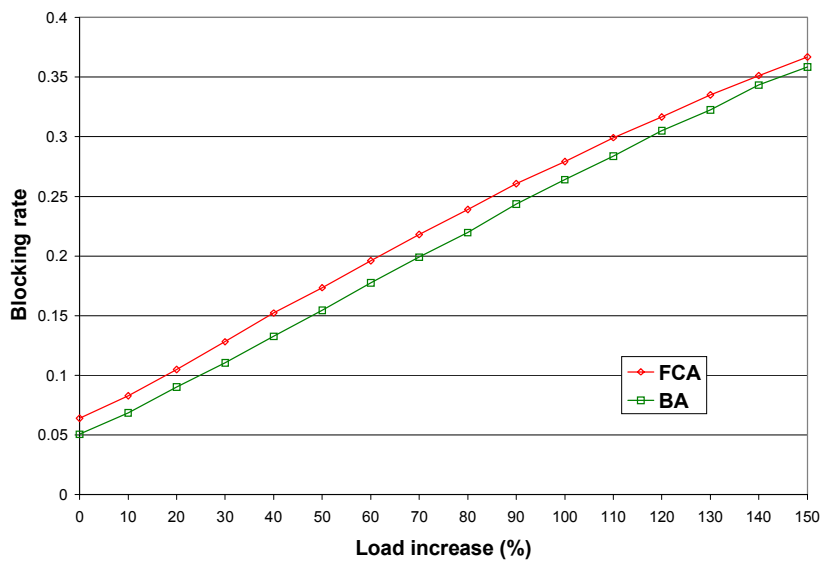

Fig. 2 Blocking rate of distributed borrowing algorithm versus FCA

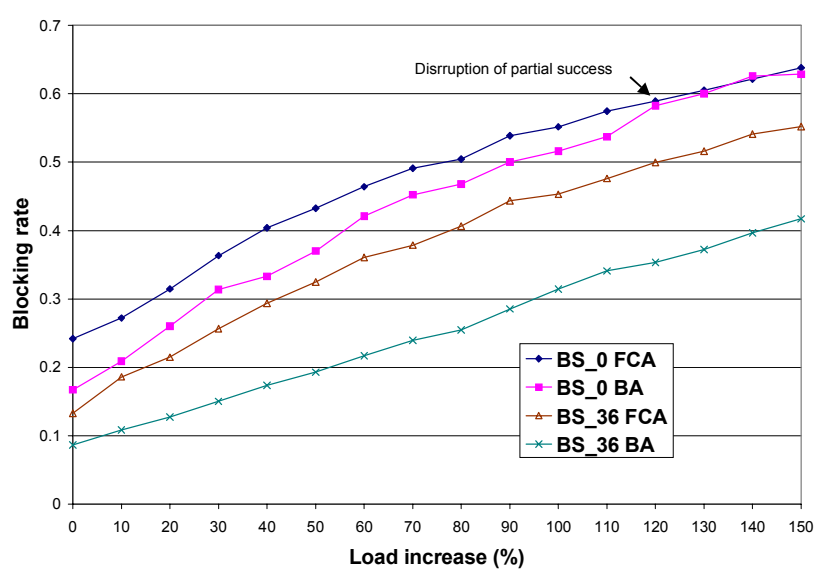

Fig. 3 Blocking rate comparison between BA and FCA for cells 0 and 36 
It is noticeable that as the load of the network increases, the performance of BA approaches that of FCAs. Because a heavily loaded network will not have spare channels that can be borrowed, running the algorithm in these traffic conditions represents only overhead for the network. Those fully reactive algorithms proposed in the literature are not able to detect the drop in their efficiency under different traffic conditions. It is clear that in order to avoid wasting signalling resources, base stations must detect these situations and decide on alternative ways to improve the efficiency of the algorithm or stop it to avoid signalling overhead.

In D-LBSB, the borrowing algorithm is not executed every time a call or handoff request is made and there are no more available channels to accommodate the request: it is triggered before the nominal channels are all used, once $h$ is reached. Moreover, it does not get only one channel, but a certain number of channels (X), the actual number depending on the average traffic load of the whole network. In our implementation, each run of the algorithm can be successful (all X channels are borrowed), partially successful (when some channels are borrowed, but not the number expected) or unsuccessful. Failure can be for one of three reasons:

- Failure type 1: there were lenders but channel locking was not possible;

- Failure type 2: all possible lenders are hot;

- Failure type 3: the network is so heavily loaded that X is zero.

There is no mention in [6] about the criteria for successive runs of the algorithm by the same borrowing cell. Our decision was that the algorithm should be re-executed if the cell reaches the threshold again, even if it has not given back all borrowed channels. If the algorithm has partial success or failure, it will not be allowed to re-execute the algorithm immediately, but it will wait for a certain time depending on the cause of the failure. The introduction of these delays is to avoid continual repetition of the algorithm, which would substantially increase the signalling load to no avail.

Table 1 shows the results of running the BA over a 24 hour simulation time.

TABLE 1

\section{RESULTS OF BORROWING ATTEMPTS}

\begin{tabular}{lllllll}
\hline $\begin{array}{l}\text { Load } \\
\text { increa- } \\
\text { se }(\%)\end{array}$ & $\begin{array}{l}\text { Total } \\
\text { number } \\
\text { of BA } \\
\text { runs }\end{array}$ & $\begin{array}{l}\text { BA } \\
\text { runs } \\
\text { with } \\
\text { success }\end{array}$ & $\begin{array}{l}\text { BA } \\
\text { runs } \\
\text { with } \\
\text { partial } \\
\text { success }\end{array}$ & $\begin{array}{l}\text { BA } \\
\text { failure } \\
\text { type 1 }\end{array}$ & $\begin{array}{l}\text { BA } \\
\text { failure } \\
\text { type 2 }\end{array}$ & $\begin{array}{l}\text { BA } \\
\text { failure } \\
\text { type 3 }\end{array}$ \\
\hline 0 & 6160 & 1994 & 1732 & 1133 & 1301 & 0 \\
50 & 5187 & 1077 & 603 & 1694 & 1813 & 0 \\
100 & 4523 & 663 & 39 & 1437 & 2371 & 13 \\
150 & 3464 & 191 & 12 & 584 & 1432 & 1245 \\
\hline
\end{tabular}

The algorithm has good performance at base load, with $40 \%$ failure. With an increase in load of $50 \%$, there is a $57 \%$ failure rate, rising to $85 \%$ at $100 \%$ increase. It should also be noted that our policy of introducing a delay reduces the number of attempts to borrow as the load increases, so saving in needless signalling messages.

It is clear that a surveillance process in the base station could recognise the conditions when the algorithm is not being efficient and avoid redundant signalling. More detailed analysis of the results (see arrow in Fig. 3) show that up to approximately $100 \%$ load increase, the algorithm gets partial success throughout the duration of the simulation run. This means that, overall, resources are available to reduce congestion, but the reactive algorithm is not accessing them in an efficient way. To improve the performance and efficiency, we propose a multi-agent system and a particular agent architecture that allows base stations be more flexible and intelligent, negotiating and co-operating with others to improve the efficiency of the channel assignment scheme. This allows the approach to including planning to attempt to balance the load in advance of reactive requests.

\section{The Proposed Multi-Agent System}

It was shown above that reactive approaches, while necessary, are not sufficient for control of resources: the base stations must have an adaptive behaviour, not only to improve their own channel usage, but also to co-operate with other base stations to improve the performance of the whole network. The inherent features of distributed problem solving make a multi-agent system a suitable approach to control such a complex system.

A multi-agent system (MAS) can be defined as a group of agents with specific roles in an organisational structure [9]. The agents interact with the environment and with each other in a co-ordinated way, as collaborators or competitors, seeking to fulfil the local or global aims of the organisation. The definition of an agent and the main characteristics that distinguish agents from other software systems can de found in [10]. Agent architectures are classified by the degree of reasoning incorporated by the agent, from a completely logical model (known as Beliefs, Desires, Intentions (BDI) architectures) to a fully reactive model with no symbolic representation.

Logical models present better behaviour than reactive ones, but they can be too slow for real time applications. Hybrid architectures combine features of logical and reactive models and are, therefore, more suitable for real time applications. The INTEgration of Reactive behaviour and Rational Planning architecture (INTERRAP) [9] is a hybrid agent architecture that also incorporates mechanisms for coordination and co-operation among autonomous agents. The INTERRAP architecture consists of a set of hierarchical layers, a knowledge base that supports the representation of different abstraction levels of knowledge and a well defined control architecture that ensures coherent interaction among layers. It was designed to react to unexpected events, to long term actions based on goals and to cope with other agent interactions. INTERRAP was, therefore, chosen as our model for agent implementation. The architecture illustrated in Fig. 4 was adapted from [9].

In our scenario, the cellular network has one agent per cell. The world interface presented to the agent includes the sensor section responsible for the perception of the environment, which would include requests for channel allocation from new calls, handoff requests, borrowing channel requests and orders for locking channels.

The communication section handles message exchanges for channel management and in the negotiation process. The action section is responsible for all execution tasks that actually allocate, release, re-allocate, lock, lend channels, manage normal and supervised handoffs and terminate appropriately unsuccessful requests. 
In the knowledge base, the world model contains the environment information and everything necessary for the operation of the reactive layer. The mental model contains the complete information about the agent, about the use of frequency channels and possibly history of traffic load in the cell. Finally the social model has relevant information about other agents' data.

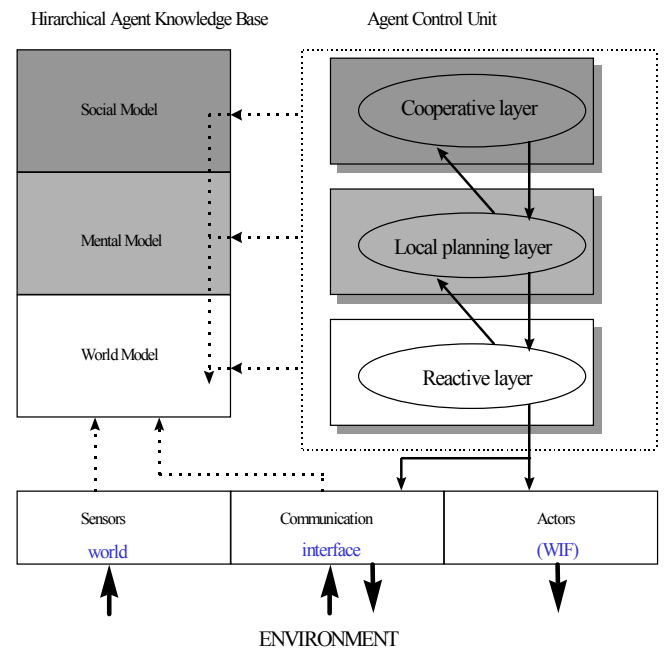

Fig. 4 The architecture of the agent

The control unit is structured to include a reactive control layer that is responsible for fast accommodation of traffic demand, a local planning control layer using other strategies to optimise the local load distribution of channels and the co-operative control layer, responsible for load balancing across a larger area. The agents follow a collaborative strategy using contract-net protocol [11]. A brief description of each layer implementation is given below:

The reactive layer uses an algorithm based on D-LBSB without channel re-assignment.

The local planning layer: determines the departing region based on the signal/noise ratio and traffic history conditions, independently of the execution of the borrowing algorithm (a difference from the purely reactive algorithm). This layer is responsible for the channel re-assignment scheme. In order to optimise local performance, the re-allocation scheme takes into account the efficiency of channel usage and traffic history

Finally the co-operative layer starts a two-phase resource negotiation when the traffic load of the compact pattern does not allow efficient performance of the channel allocation scheme. The agent in the hot cell, using the contract-net protocol, requests its co-channel cells to make offers for moving calls to their regions. To be able to make a bid, the co-channel cells need to compute a utility function that requires the channel availability of its compact pattern and the percentage of users in the selected departing areas. Therefore, they request this information to each cell of their compact pattern. Receiving all the information, the cochannel cells compute the utility function and send the result (as a bid) to the requesting hot cell. The requesting hot cell chooses the best bid and sends an acknowledgment to the winning co-channel cell. This ends the first phase of the resource negotiation. The second phase consists of a joint plan engaged by the compact pattern of the winning cochannel cell, the neighbouring cells of the hot cell which make borders with the chosen compact pattern and the hot cell itself. For the execution of the joint plan, the hot cell is the manager, and the other cells are the contractors. The task to be executed by the contractors is to ask the mobiles stations in the selected departing areas to verify the quality of the reception of the control channel of the closest neighbouring cell. If the quality of the reception is good enough, the base station will request a management handoff to the neighbouring cell with best transmission quality and the mobile possibly will be moved to that cell. However, not all contractors will necessarily request management handoffs at the same time; in fact, some contractors might not request management handoffs at all, serving only as recipients of mobile users. The decision of whom and when to perform the task is dependent on the economic model used by the manager of the plan. Finally, the assessment of the plan and the load conditions of the network will indicate the termination of the joint plan.

We are currently implementing the multi-agent system in OPNET $^{\mathrm{TM}}$ in the same simulation context as FCA and BA. Simulation results will allow investigating the performance and flexibility that can be achieved by the multi-agent system in comparison with a pure reactive approach.

\section{CONCLUSIONS}

This paper demonstrates the deficiencies of fully reactive approaches in channel assignment for mobile networks and proposes a new strategy to improve the performance and flexibility of channel allocation by using intelligent cooperative agents.

\section{ACKNOWLEDGMENT}

This research is being sponsored by the Brazilian Federal Agency for Post-Graduate Education (CAPES) and the Federal Centre of Technologic Education of Parana' (CEFET-PR Brazil) where the author is a lecturer.

\section{REFERENCES}

[1] S. M. Elnoubi, R. Singh and S. C. Gupta, "A new frequency channel assignment algorithm in high capacity mobile communication systems," IEEE Transactions on Vehicular Technology, vol. 31, no. 3, pp. 125-131, August 1982.

[2] M. Zhang and T.-S. P. Yum, "Comparisons of channel assignment strategies in cellular mobile telephone systems," IEEE Transactions on Vehicular Technology, vol. 38, no. 4, pp. 211-215, November 1989.

[3] J. Karlsson and B. Eklundh, "A cellular mobile telephone system with load sharing - an enhancement of directed retry," IEEE Transactions on Communications, vol. 37, no. 5, pp. 530-535, May 1989.

[4] H. Jiang and S. S. Rappaport, "CBWL: A new channel assignment and sharing method for cellular communication systems," IEEE Transactions on Vehicular Technology, vol. 43, no. 2, pp. 313-322, May 1994. 
[5] S. K. Das and S. K. Sen and R. Jayaram, "A dynamic load balancing strategy for channel assignment using selective borrowing in cellular mobile environment," Wireless Networks, vol. 3, pp. 333-347, 1997.

[6] S. K. Das and S. K. Sen, R. Jayaram and P. Agrawal, "A distributed load balancing algorithm for the hot cell problem in cellular mobile networks," Proceedings of the $19976^{\text {th }}$ IEEE International Symposium on High Performance Distributed Computing, pp. 254-263, Portland, USA, August 5-8 1997.

[7] I. Katzela and M. Naghshineh, "Channel assignment schemes for cellular mobile telecommunication systems: a comprehensive survey," IEEE Personal Communications Magazine, vol. 3, no. 3, pp. 10-31, June 1996.
[8] V. H. MacDonald, "Advanced mobile phone services: the cellular concept," Bell Systems Technology Journal, vol. 58, no. 1, pp. 15-41, January 1979.

[9] J. P. Müller, "The design of intelligent agents: a layered approach," Lecture Notes in Artificial Intelligence 1177, Subseries of Lecture Notes in Computer Science, Edited by J. Carbonnel and J. Siekmann, Springer, 1996.

[10] E. L. Bodanese and L. Cuthbert, "Distributed channel allocation scheme for cellular networks using intelligent agents," $7^{\text {th }}$ International Conference in Telecommunication Systems, pp. 156-165, Nashville TN, USA, March 18-21 1999.

[11] R. G. Smith, "The contract net protocol: high-level communication and control in a distributed problem solver," In Readings in Distributed Artificial Intelligence. Morgan Kaufman Publishers, Bond, A. and Gasser, L. editors, 1988. 